\title{
A Hands-Off Physical Therapy Assistance Robot for Cardiac Patients
}

\author{
Kyong Il Kang, Sanford Freedman and Maja J Matarić \\ Interaction Laboratory \\ Computer Science Department \\ University of Southern California \\ 941 West 37th Place \\ Los Angeles, CA 90089-0781 \\ \{kyongkan |sfreedma|mataric\}@usc.edu
}

\author{
Mark J. Cunningham and Becky Lopez \\ Department of Cardiothoracic Surgery \\ Keck School of Medicine \\ University of Southern California \\ 1510 San Pablo Street, Suite 415 \\ Los Angeles, CA 90033-4612 \\ \{mcunningham|bmlopez\}@surgery.usc.edu
}

\begin{abstract}
This paper presents a feasibility study of using socially-aware autonomous robots to assist hospitals in reducing the effects of nursing shortages. A hands-off assistive robot is described that provides motivation and support for cardiac patients who must perform regular but painful breathing exercises. Initial validation of the system has garnered positive responses from test subjects and shows that robots have a potential to aid nursing staff in some tasks requiring patient interaction.
\end{abstract}

\section{INTRODUCTION}

Registered nurses play a vital role in patient care. Unfortunately, the ongoing nursing shortage affecting medical institutions nation-wide is expected to worsen in the future [1]. The population of the United States is aging, with a predicted upcoming increase of 36 million people over 65 between 2000 and 2030 [2]. At the same time, the registered nurses community is also aging; the percentage of female nurses over the age of 35 is on the rise while fewer young people are entering the field of nursing than in the past [3]. Nursing shortages are not specific to the United States, but are a world-wide problem [4]. Reduced nurse staffing results in each nurse assuming additional responsibilities and an increased work volume, sometimes including mandatory overtime. Each additional patient can increase the chance of nurse burnout by $23 \%$ and job dissatisfaction by $15 \%$. The resulting nurse turnover is not only financially draining $(\$ 42,000$ for a hospital medical and surgical general unit and $\$ 64,000$ for a specialty nurse), but compounds the overall shortage problem. Additionally, the lives and well-being of patients are directly affected. Recent studies have shown that each patient added to a nurse's workload increases the chance of patient mortality by $7 \%$ [1]. Furthermore, the frequency and severity of non-mortal complications rise with additional nurse workload [5].

The work presented in this paper aims to address the nurse shortage problem through the development of sociallyaware assistive robots capable of freeing nurses of selective repetitive and time-consuming patient interactions. By providing robotic assistance to patients, nurses are allowed greater flexibility in directing their efforts. We present an autonomous robotic system capable of assisting nurses by helping cardiac patients in a repetitive, time-consuming and often painful breathing (spirometry) exercise.

\section{RELATED WORK}

Assistive robotics focuses on aiding those in need, including patients, the elderly, and individuals with disabilities. As a robot task domain, it has received growing attention in the past couple of decades [6]. Most relevant to our research, assistive robotics can be divided into two distinct categories: 1) hands-on non-interactive assistive devices/robots and 2) hands-off interactive assistive robots.

The hands-on assistive devices and robots have been developed using a variety of approaches. The ProVAR project [8], originating from the DeVAR project [7], developed a fixed robotic workstation to assist in vocational activities. MIME [9] and MIT-Manus [10] also employed manipulatormounted fixed robots to assist stroke patients during physical therapy. The recovery of stroke-afflicted limbs requires numerous repetitive exercises; these robots help patients by physically supporting their limbs with mechanical manipulators, allowing for increased time spent in physical therapy. Manipulator-mounted wheelchair robots are another active area of rehabilitation robotics [11], [12]. In general, this family of hands-on assistive robotics typically (but not exclusively) aids patient mobility primarily through physical contact and with minimal if any social interaction.

In contrast, the hands-off assistive robotics approach minimizes safety concerns by avoiding, and in many cases entirely eliminating, physical contact with patients. HelpMate [13], a commercially available mobile robot, autonomously navigates hospital floors delivering medicine and supplies to hospital staff and meal trays to patients. Pearl the NurseBot [14], interactively assists the elderly by providing daily reminders and navigation guidance. Pearl's appearance was designed to facilitate interaction and minimize intimidation. Engineered to help the elderly and the handicapped, Care-OBot [15] provides assistance with household tasks, such as fetching objects and is capable of acting as a communications platform, allowing patients to watch TV or call a doctor through interaction with a speech interface or a touch-screen.

In contrast to both categories above, few hands-off robots to date have been developed to provide medical patients with physical therapy. In the work we propose, the safety advantages of hands-off assistive robotics are used in combination with social interaction, to enable a mobile robot, Clara, to 
serve as a practical physical therapy assistant and helping patients recover from cardiac surgery.

\section{APPROACH}

\section{A. General Philosophy and Task}

It is well established that people engage with physically embodied creatures, even simple ones, and ascribe life-like properties to them [16]. Our work aims to leverage those tendencies through the use of assistive robots with three specific traits: social awareness, autonomy, and the absence of tactile input or physical manipulation. Social awareness allows the robots to perform tasks that involve social interaction with patients. Autonomy frees up nurses' time but requires the robot to be able to complete tasks unassisted as well as collect, store, and report data to medical staff for subsequent analysis and diagnosis. The hands-off nature of the system allows the other two components, autonomy and social awareness, to be performed safely and therefore more readily introduced into hospital environments.

The work described in this paper is a feasibility study aimed at determining the acceptance of a system (implemented as a basic research prototype) with the above capabilities, addressing breathing (spirometry) exercises as the target assistive task. Cardiac patients post-surgery need to perform regular lung exercises in order to prevent infection and facilitate recovery. In these workouts, normally assisted by a cardiac nurse, patients are asked to use a spirometer, a small plastic device designed to measure the amount and velocity of inhaled air. Patients slowly inhale to a predetermined volume, which is increased as recovery progresses. Through this activity, the patent's lungs are forced to fully expand and contract, providing exercise and preventing pneumonia. For maximum benefit, spirometry exercises must be performed ten times per hour for several days, in some cases weeks, after cardiac surgery. While technical skill in using the spirometer and instruction in its proper use are required in the first couple of sessions, the vast majority of the remaining sessions require only monitoring and motivation. We developed Clara, shown in Fig. 1, an autonomous assistive robot that encourages and monitors spirometry exercises.

\section{B. Evaluation Testbed}

Clara is constructed from standard off-the-shelf components. Mobility and primary battery power are provided by an ActivMedia Pioneer $2 \mathrm{DX}$ mobile robot platform. A $1 \mathrm{GHz}$ Pentium III computer running Gentoo Linux resides inside, controlling the motors and providing basic I/O support. Sensors on the robot include a laser range-finder, a camera, and a microphone. The laser, a SICK LMS200, provides a $180^{\circ}$ field of view used for obstacle avoidance and localization; it is mounted so as to be able to detect the feet of hospital beds. The vision system consists of a Sony EVI-D30 pan-tiltzoom camera with a $640 \times 480$ frame-grabber. The produced video signal is fed through to an ACTS color-tracking server, which provides color blob detection. Acoustic perception is generated with a Shure 503BG unidirectional dynamic microphone designed for computer voice recognition. The

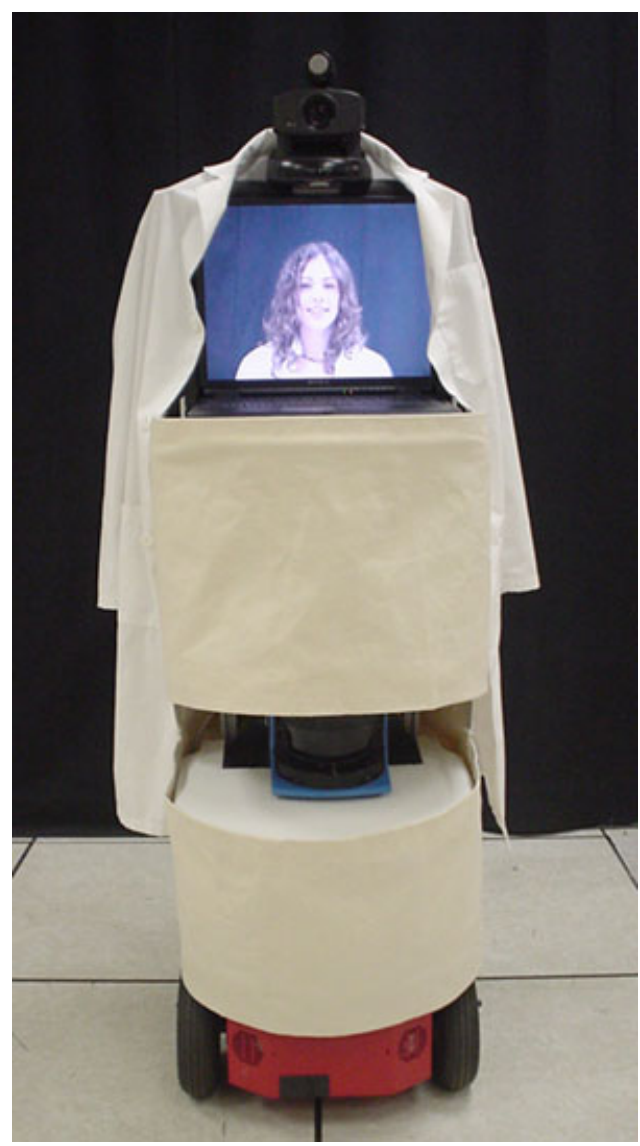

Fig. 1. An image of Clara

microphone has a frequency response of $100 \mathrm{~Hz}$ to $7 \mathrm{kHz}$, and is amplified by a Rolls Personal Monitor Amp+ and sent through the sound card (in the base of the robot) running the CMU Sphinx 2 voice recognition engine. Clara uses a standard unpowered computer speaker to communicate with the user, and plays pre-recorded female speech. A $2 \mathrm{GHz}$ Linux laptop is used to run the control and interaction algorithm and its 15" screen for displaying Clara's face using MPlayer video playback application. Player [17], a robot device server, is used for inter-component communication. The physical components are mounted on a custom-built 54" chassis.

\section{Control Approach}

Clara's control architecture sequences behaviors that handle navigation, patient interaction, and spirometer tracking. To simplify localization and navigation in the hospital room, and to maximize safety, a sheet of laser-reflective material was taped to each hospital bed. Fig. 2 shows the hospital room environment we constructed in our lab, which was based on actual rooms at the USC University Hospital cardiac ward, where Clara is currently being tested.

Clara's control system consists of four capabilities/behaviors: patient locating, patient interaction, spirometer tracking, and exiting the hospital room. Fig. 3 overviews Clara's control flow. Locating the patient begins with wall 


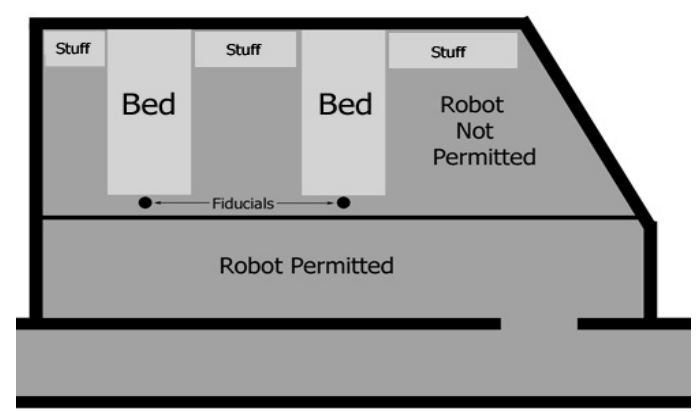

Fig. 2. Example hospital room set-up

following along the hallway, through the room door, and up to the patient's bed, where the reflective fiducial is detected. Next, the interaction behavior takes over and Clara asks the patient if $\mathrm{s} / \mathrm{he}$ is willing to perform the spirometry exercise. If the answer is yes (detected either through a speech interface or with a color marker), Clara asks if instructions for using the spirometer are needed, and provides them if requested or proceeds directly to the exercise. Clara then requests that the patient hold the spirometer and when the spirometer is detected, the spirometer tracking behavior is instantiated. Using color markers on the spirometer, detected by the color blob-tracking server, the patient's breaths are counted and recorded until the spirometer is removed from Clara's field of view. At that point, the exercise is finished; Clara thanks the patient and the control system enters the final component, returning to the starting position. This is achieved by reversing its path and executing a (opposite side) wall following behavior. Collected patient data, including breathing results and willingness to perform the exercise, are available to the hospital staff and can be reported at selected intervals.

\section{EVALUATION}

The first step in validating the feasibility and effectiveness of the above-described assistive robot involved experiments with healthy test subjects. Those experiments were performed in an emulated hospital room in the USC Interaction Laboratory, and supervised by expert cardiac ward staff. Five USC students served as experimental subjects; they wore hospital gowns and used authentic hospital spirometers modified only with the color patches for ease of spirometer tracking (described above). After the experiments, the subjects were asked to complete a questionnaire reporting their impressions of the robot. Fig. 4 shows the robot interacting with a subject; videos of the experiments can be found at http://robotics.usc.edu/interaction/?l= Research:Projects: Spirometry: index.

The experiments showed that all of Clara's control components performed successfully. The speech recognition system failed to recognize the voice of one of the five test subjects due in part to the acoustics of the laboratory environment, moderate background noise, and the softness of the voice. Fortunately, when Clara was subsequently tested in an actual
TABLE I

Questionnaire Results Of The Five Test Subjects

\begin{tabular}{|l|l|l|l|}
\hline Questions & A lot & Somewhat & No \\
\hline $\begin{array}{l}\text { Is robotic assistance of spirometry exer- } \\
\text { cise a good idea? }\end{array}$ & 3 & 2 & 0 \\
\hline Did the robot assist your exercise? & 1 & 3 & 1 \\
\hline Did you enjoy your assisted exercise? & 1 & 4 & 0 \\
\hline Did you like interacting with the robot? & 3 & 2 & 0 \\
\hline
\end{tabular}

hospital room, the speech recognition system performed successfully. Proper tuning is critical for optimal performance.

Table I summarizes the data collected from the patient questionnaires. All subjects were interested in the robotassisted spirometry exercise and enjoyed the interaction with Clara. The subjects also provided overall satisfaction ratings reflecting how helpful they considered the robot to be in assisting in their spirometry exercise. The average overall satisfaction rating was $84.6 \%$.

The subjects provided the following suggestions about improving the system: 1) an elaboration of the instructions, so they could better understand how to perform their spirometry exercise while assisted by Clara; 2) improvement of the speech recognition system; and 3) use of richer interaction, involving more interactive conversation. We are currently addressing all of these issues, and others, as discussed in the next section.

\section{CONTINUING AND FUTURE WORK}

Based on user feedback resulting from the experiments and experiences with Clara, considerable work still remains. Clara's user input subsystems, mainly voice recognition, must be further developed to improve accuracy and reduce patient strain. Safety also remains a primary issue. Hospitals are highly dynamic environments that can hinder the robot's ability to perform obstacle detection and avoidance. A more comprehensive system for collision avoidance will be incorporated.

Most relevant to our research interests, Clara will be equipped with a richer set of social behaviors. We will experiment with different means of patient motivation, through

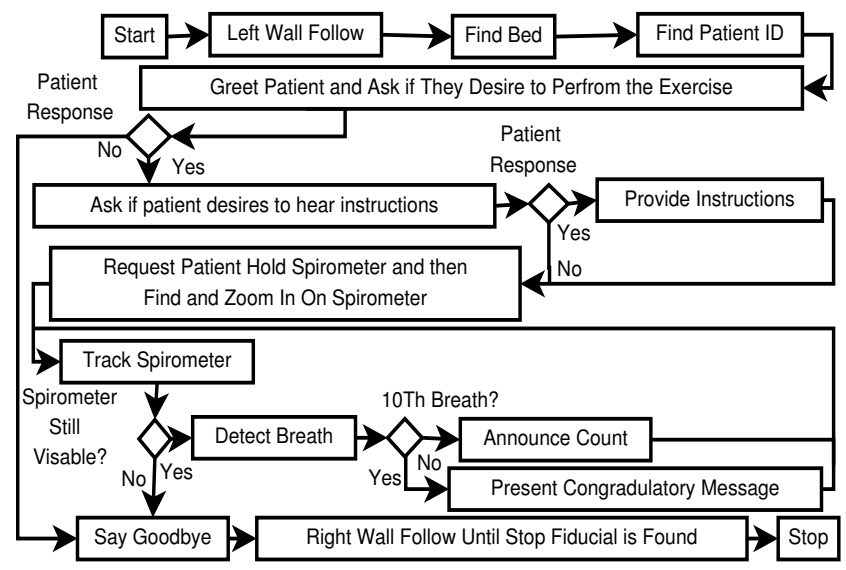

Fig. 3. Control flow of Clara's architecture 


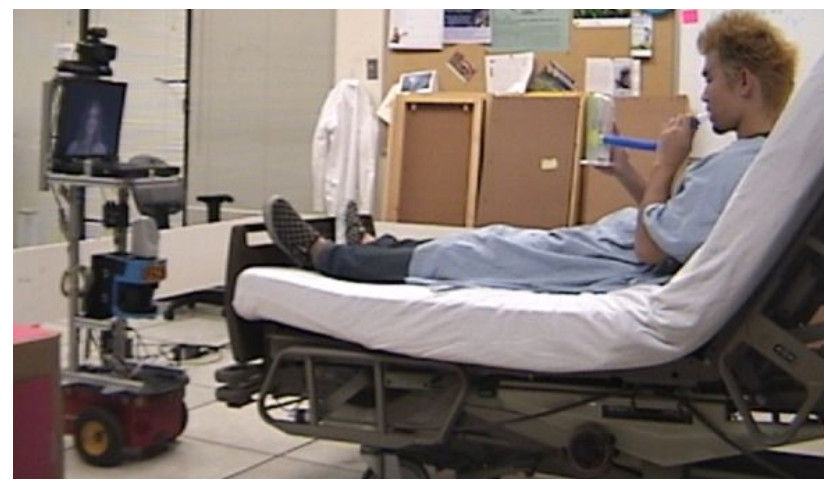

Fig. 4. Clara (without covers) assisting a patient with the spirometry exercise

the use of verbal games, sounds, and robot movement. Furthermore, alterations to the physical appearance of the robot will be investigated as an additional path toward patient motivation and engagement.

While the above directions will focus on patient interaction, further work will also be undertaken to improve Clara's cost-effectiveness and direct effect on nurse efficiency. This particular study focuses on robot-assisted spirometry, but we envision that such robots can be endowed with a diverse set of functionalities. For example, if the robot is equipped with appropriate sensors to detect patient posture, it can also report any undetected emergencies, such as a fallen patient. In addition, an accessible database of patient performance and status can be developed in combination with increased navigational abilities and task queueing. This will avoid the need of having nurses direct the robot to specific patient rooms.

Concurrent and future research being performed by the Interaction Laboratory will also study the differences in patients' responses to and relative effectiveness of different types of interaction media. Devices to be analyzed include computer/television screens, personal digital assistants, and even simple voice recordings.

\section{CONCLUSION}

This paper has suggested a means of reducing nurse workload through the use of autonomous socially-aware robots. Initial feasibility of this approach has been demonstrated with Clara, a robot that assists patients in repetitive spirometry exercises.

The described work aims at two related but distinct goals: 1) automation of assistive tasks and 2) improvement of patient outcomes. In this particular case, we aimed to provide help to the nursing staff, at the same time as improving the frequency and quality of the patient's breathing exercises. The evaluation of the success of each of the goals will require further in-depth experiments; the second goal will also require a more clinical emphasis.

We hope that, through further development, additional tasks well suited for assistive robotics will be identified, help- ing to increase nurse efficiency. We do not advocate or aim to replace hospital nurses, whose expertise is irreplaceable, but instead to help them to focus their time and resources, and improve patient well-being, through the use of assistive interactive robotics.

\section{ACKNOWLEDGMENTS}

This work was supported by the USC Provost's Center for Interdisciplinary Research and by the Okawa Foundation.

\section{REFERENCES}

[1] L. H. Aiken, S. P. Clarke, D. M. Sloane, J. Sochalski, and J. H. Silber, Hospital Nurse Staffing and Patient Mortality, Nurse Burnout, and Job Dissatisfaction, The Journal of the American Medical Association, vol. 288, no. 16, 2002, pp. 1987-1993.

[2] M. R. Goulding, M. E. Rogers, and S. M. Smith, Public Health and Aging: Trends in Aging - United States and Worldwide, The Journal of the American Medical Association, vol. 289, no. 11, 2003, pp. 13711373.

[3] P. I. Buerhaus, D. O. Staiger, and D. I. Auerbach, Implications of an Aging Registered Nurse Workforce, The Journal of the American Medical Association, vol. 283, no. 22, 2000, pp. 2948-2954.

[4] J. Spetz, and R. Given, The Future Of The Nurse Shortage: Will Wage Increases Close The Gap?, Health Affairs, vol. 22, no. 6, 2003, pp. 199-206.

[5] J. Needleman, and P. Buerhaus, Nurse staffing and patient safety: current knowledge and implications for action, International Journal for Quality in Health Care, vol. 15, no. 4, 2003, pp. 275-277.

[6] M. Hillman, Rehabilitation robotics from past to present - a historical perspective, in Proceedings of the International Conference of Rehabilitation Robotics 2003, Kaist, Daejeon, Korea, April 2003, pp. 1-4.

[7] H.F.M. Van der Loos, J. Hammel, L.J. Leifer, DeVAR transfer from R\&D to vocational and educational settings, in Proceedings of the Fourth International Conference on Rehabilitation Robotics, Wilmington, Delaware, June 1994, pp. 151-156.

[8] J. J. Wagner, M. Wickizer, H. F. M. Van Der Loos, L. J. Leifer, User testing and design iteration of the ProVAR user interface, in 8th IEEE International Workshop on Robot and Human Interaction, Pisa, Italy, September 1999, pp. 18-22.

[9] P. C. Shor, P. S. Lum, C. G. Burgar, H. F. M. Van Der Loos, M. Majmundar, and R. Yap, The effect of Robotic-Aided therapy on upper extremely joint passive range of motion and pain, Integration of Assistive Technology in the Information Age, 2001, pp. 79-83.

[10] H. I. Krebs, B. T. Volpe, M. L. Aisen, W. Hening, S. Adamovich, H. Poizner, K. Subrahmanyan, N. Hogan, Robotic applications in neuromotor rehabilitation, Robotica, vol. 21, no. 1, 2003, pp. 3-12.

[11] M. Hillman, K. Hagan, S. Hagan, J. Jepson, and R. Orpwood, A Wheelchair Mounted Assistive Robot, in Proceedings of the International Conference on Rehabilitation Robotics, Stanford, California, USA, July 1999, pp. 86-91.

[12] R. M. Mahoney, The Raptor Wheelchair Robot System, Integration of Assistive Technology in the Information Age, 2001, pp. 135-141.

[13] B.Krishnamurthy, J. Evans, HelpMate: A robotic courier for hospital use in Proceedings of the IEEE International Conference on Systems, Man and Cybernetics, Vol. 2, 1992, pp. 1630-1634.

[14] M. Montemerlo, J. Pineau, N. Roy, S. Thrun, and V. Verma, Experiences with a Mobile Robotic Guide for the Elderly, in Proceedings of the AAAI National Conference on Artificial Intelligence, Edmonton, Canada, 2002, pp. 587-592.

[15] B. Graf, M. Hans, J. Kubacki, and R. D. Schraft Robotic home assistant Care-O-bot II, in Proceedings of the Second Joint EMBS/BMES Conference, vol. 3, Houston, Texas, USA, October 2002, pp. 23432344.

[16] B. Reeves, and C. Nass, The Media Equation: How People Treat Computers, Television, and New Media Like Real People and Places, Cambridge University Press, New York, NY, 1998.

[17] R. T. Vaughan, B. Gerkey, and A. Howard, On Device Abstractions For Portable, Resuable Robot Code, in Proceedings of the IEEE/RSJ International Conference on Intelligent Robot Systems, Las Vegas, Nevada, USA, October 2003, pp. 2121-2427. 